\title{
p130Cas is an essential transducer element in ErbB2 transformation
}

\author{
Sara Cabodi,*,1 Agata Tinnirello,*,1 Brigitte Bisaro,*,1 Giusy Tornillo,* \\ Maria del Pilar Camacho-Leal,* Guido Forni,* Rodica Cojoca,* Manuela Iezzi, ${ }^{\$}$ \\ Augusto Amici, Maura Montani," Alessandra Eva, ${ }^{\dagger}$ Paola Di Stefano,* \\ Senthil K. Muthuswamy, ${ }^{\ddagger}$ Guido Tarone, ${ }^{*}$ Emilia Turco, ${ }^{*}$ and Paola Defilippi ${ }^{*, 2}$ \\ *Molecular Biotechnology Center, University of Torino, Turin, Italy; ${ }^{\dagger}$ Laboratorio di Biologia \\ Molecolare, Istituto G. Gaslini, Genoa, Italy; ${ }^{\ddagger}$ Cold Spring Harbor Laboratory, Cold Spring Harbor, \\ Massachusetts, USA; ${ }^{\$}$ Center for Aging Sciences (CESI) Aging Research Center, G. D'Annunzio \\ University, Chieti, Italy; and ${ }^{\|}$Department of Molecular, Cellular, and Animal Biology, University of \\ Camerino, Camerino, Italy
}

ABSTRACT The ErbB2 oncogene is often overexpressed in breast tumors and associated with poor clinical outcome. p130Cas represents a nodal scaffold protein regulating cell survival, migration, and proliferation in normal and pathological cells. The functional role of p130Cas in ErbB2-dependent breast tumorigenesis was assessed by its silencing in breast cancer cells derived from mouse mammary tumors overexpressing ErbB2 (N202-1A cells), and by its reexpression in ErbB2-transformed p130Cas-null mouse embryonic fibroblasts. We demonstrate that p130Cas is necessary for ErbB2-dependent foci formation, anchorage-independent growth, and in vivo growth of orthotopic N202-1A tumors. Moreover, intranipple injection of p130Cas-stabilized siRNAs in the mammary gland of Balbc-NeuT mice decreases the growth of spontaneous tumors. In ErbB2-transformed cells, p130Cas is a crucial component of a functional molecular complex consisting of ErbB2, c-Src, and Fak. In human mammary cells, MCF10A.B2, the concomitant activation of ErbB2, and p130Cas overexpression sustain and strengthen signaling, leading to Racl activation and MMP9 secretion, thus providing invasive properties. Consistently, p130Cas drives N202-1A cell in vivo lung metastases colonization. These results demonstrate that p130Cas is an essential transducer in ErbB2 transformation and highlight its potential use as a novel therapeutic target in ErbB2 positive human breast cancers.-Cabodi, S., Tinnirello, A., Bisaro, B., Tornillo, G., Camacho-Leal, M. P., Forni, G., Cojoca, R., Iezzi, M., Amici, A., Montani, M., Eva, A., Di Stefano, P., Muthuswamy, S. K., Tarone, G., Turco, E., Defilippi, P. p130Cas is an essential transducer element in ErbB2 transformation. FASEB J. 24, 000-000 (2010). www.fasebj.org

Key Words: breast cancer $\cdot$ tumorigenesis $\cdot$ invasion $\cdot$ metastasis $\cdot$ signaling

The ErbB2 oncogene (also known as HER2) belongs to the epidermal growth factor-receptor family, and its overexpression and activation have been detected in a large subset of mammary cancers $(1,2)$. Molecular and clinical studies indicate that ErbB2 has important implications in tumor etiology and progression. Activated ErbB2 stimulates many intracellular signaling molecules, including the mitogen-activated protein kinase, Src kinase, and phospholipase C gamma (3).

p130Cas was originally identified as a tyrosine phosphorylated protein on transformation by v-Src and v-Crk oncogenes and for its ability to associate with Crk $(4,5)$. p130Cas structural motifs and its post-translational modifications enable mechanical extension (6) and interactions with many proteins leading to multiprotein complexes (7-9). In normal cells, p130Cas modulates cell motility, survival, and proliferation (10). In particular, c-Src-mediated p130Cas phosphorylation and the assembly of a p130Cas-Crk-DOCK180 scaffold drive cell migration and responses to mechanical stress (11-13).

p130Cas is emerging as an important player in the transformation and invasion driven by different oncogenes. Its involvement in c-Src-mediated tumorigenesis has been demonstrated by the inability of c-Src to transform p130Cas-null MEFs (14). It has also been reported recently that knockdown of p130Cas causes proliferative arrest in breast cancer cell lines harboring oncogenic mutations in KRAS, BRAF, PTEN, and PIK3CA (15), underlying a role for p130Cas as a general regulator of breast cancer cell growth induced by different oncogenes.

We have previously shown that double-transgenic mice originated by crossing MMTV-p130Cas and MMTV-NeuT mice, which express the oncogenic form of the rat neu gene, homologous to human ErbB2 $(16,17)$, showed an accelerated onset of mammary tumor formation (10). In addition, the analysis of human breast samples revealed that tumors

\footnotetext{
${ }^{1}$ These authors contributed equally to this work.

${ }^{2}$ Correspondence: Molecular Biotechnology Center, University of Torino, Torino, Via Nizza 52, 10126 Torino, Italy. E-mail: paola.defilippi@unito.it

doi: 10.1096/fj.10-157347
} 
overexpressing both p130Cas and ErbB2 are characterized by an elevated proliferation index (10).

In this work, the role of p130Cas in ErbB2 transformation was assessed in epithelial cells expressing the ErbB2 proto-oncogene and closely mimicking the human pathology (N202-1A and 10A.B2). As an alternative genetically relevant model for molecular and functional studies in a p130Cas null background, mouse embryo fibroblasts (MEFs) derived from knockout animals were also used (14). Moreover, human 10A.B2 mammary epithelial cells expressing a synthetic-ligandinducible ErbB2 receptor (20) were used in 2- and 3-dimensional (2-D and 3-D) cultures. In these cells, ErbB2 activation interferes with the physiological morphogenetic process of mammary acini formation and reinitiates proliferation, disrupting cell polarity and giving rise to multiacinar, noninvasive structures, mimicking the early stages of breast cancer. By these means, we demonstrate that p130Cas is necessary for ErbB2 transformation, and is required to trigger growth and invasion of ErbB2-transformed cancer cells.

\section{MATERIALS AND METHODS}

\section{Antibody, reagents, inhibitors, and mice}

p130Cas mAbs have been previously described (10). mAbs CB11 to ErbB2 was from Novocastra Laboratories (Newcastle, UK). An additional mAb directed to the cytoplasmic domain of ErbB2 was prepared in our laboratory, by immunizing mice with a recombinant protein encompassing amino acids 1031-1160 of rat ErbB2 cDNA sequence. mAbs to Fak, GFP, Rac 1, lamininV, and vinculin were from Millipore (Billerica, MA, USA). Antibodies to c-Src, p-Tyr PY99, and actin were from Santa Cruz Biotechnologies (Palo Alto, CA, USA). Cleaved caspase 3, pTyr925 Fak, p314Fak, and pTyr416 Src antibodies were from Cell Signaling (Beverly, MA, USA). KI67 mAbs were from Novocastra Laboratories. Matrigel and collagen I were from BD Transduction Laboratories (Franklin Lakes, NJ, USA). Secondary antibodies conjugated with peroxidase were from Sigma (St. Louis, MO, USA). Secondary antibodies conjugated with FITC and TRITC were from Alexa Molecular Probes (Invitrogen, Carlsbad, CA, USA). SU6656 was from Calbiochem (San Diego, CA, USA), PT 573228 was obtained from Tocris (Ellisville, MO, USA) and Lapatinib was a kind gift of Dr. Giorgio Vállabrega (Istituto per la Ricerca e Cura del Cancro, Candiolo, Italy). Mice were from Charles River Laboratories (Calco, Italy).

\section{Cell cultures}

NIH3T3 were maintained in DMEM, 10\% CS. MEFs were cultured in DMEM, 10\% FCS, and N202-1A cells in DMEM$20 \%$ FCS. 10A.B2 cell cultures and 3-D assays were performed in agreement with protocols reported online (http://muthuswamylab.cshl.edu/ml_protocols/html). Live images were collected by using Zeiss microscopy (Carl Zeiss, Oberkochen, Germany) at $\times 20$ or $\times 63$.

\section{Generation of recombinant retroviruses and inducible lentiviruses}

FL BamHI/SalI p130Cas cDNA from pcDNA3 (10) was cloned into pBabe-GFP. pBabe-ErbB2(VE) retrovirus was described by Holbro et al. (18). MEFs or 10A.B2 cells were infected with viral particles from 293-GP packaging cells and selected with $1 \mu \mathrm{g} / \mathrm{ml}$ puromycin and $200 \mu \mathrm{g} / \mathrm{ml}$ HygromycinB (Sigma).

p130Cas-shRNA (5'GGAGGTGTCTCGTCCAATA3') and scramble-shRNA (Ctr) (5'GCCCAGCCACGGACCTTTA3') sequences were obtained by Sigma and cloned into pSuper Retro. Subcloning into pLVTHM lentiviral vectors, viral production, and N202-1A cell infection was described by Trono (19). For p130Cas reconstitution, human p130Cas cDNA was cloned into pCCL lentiviral vector, and viral particles production was performed as described above.

\section{Immunoblotting, immunoprecipitation analysis, Rac1} pulldown assay, and gelatin zymography

Protein extracts, WB, and IPs were performed as described by Cabodi et al. (20). Rac1 pulldown assay was performed as described by Di Stefano (21).

For gelatin zymography, conditioned media were collected from confluent MCF10A.B2 cells treated with AP1510 in serum-free medium for $18 \mathrm{~h}$ and processed as described by Kim et al. (22). Image J software (U.S. National Institutes of Health, Bethesda, MD, USA) was used for blot quantification.

\section{In vitro assays}

Foci formation was evaluated as described previously (23). Soft agar colonies were evaluated by seeding in triplicate on top of $0.4 \%$ agar in 6 -well plates $10^{4} \mathrm{MEF} /$ well in DMEM$10 \%$ FCS or $3 \times 10^{4}$ N202-1A/well in DMEM-20\% FCS mixed 1:1 with $0.8 \%$ agar (Sigma). To evaluate anoikis, N202.1A cells were grown in suspension for $24 \mathrm{~h}$ on dishes coated with $4 \%$ agarose in DMEM (1:1). Two-dimensional migration and invasion assays were performed as by Di Stefano et al. (21).

\section{In vivo assays}

N202-1A cells $\left(10^{5}\right)$ were injected into the fat pad of FVBNeuN mice. The incidence and growth of tumors were evaluated as described by Di Stefano (21). For the experiments of lung colonization, $10^{5}$ control, silenced, and reconstituted N202-1A cells were injected intravenously in tail veins of nude mice. Drinking water was supplemented with doxycycline $(0.1 \mathrm{mg} / \mathrm{ml})$, protected from light, and changed every 2 or $3 \mathrm{~d}$. After 5 wk, lungs were stained by India ink, and surface lung nodules were counted.

Control or p130Cas SiStable siRNA (NNUUGACUAAUAGUCUACAUUUA) (Dharmacon; Ordda, Erembodegem, Belgium) (10 $\mathrm{nM}$ in $25 \mu \mathrm{l} \mathrm{PBS})$ were injected intranipple (24) in 12 wk-old female Balbc NeuT mice $1 \times /$ wk for $5 \mathrm{wk}$. Whole-mount analyses were performed as described by Cappello et al. (25).

\section{In vitro and in vivo extravasation assays}

In vitro, $10^{5}$ human umbilical vein endothelial cells (HUVECs) stained with CellTracker blue dyes (Invitrogen) were seeded on transwells coated with $16,5 \mu \mathrm{g} / \mathrm{ml} \mathrm{FN} \mathrm{(Costar,} \mathrm{pore} \mathrm{size} 5 \mu \mathrm{m}$ ) and allowed to grow for 72 h. $10^{5}$ N202-1A cells were plated on top of HUVEC monolayer and allowed to migrate for $72 \mathrm{~h}$. Transwell bottom sides were fixed, and images were collected by using Zeiss microscopy at $\times 40$. For in vivo extravasation assay, $10^{7}$ cells stained with CellTracker red dyes (Invitrogen) were injected into the tail vein of nude mice and lungs were fixed in PFA $4 \%$ after $2 \mathrm{~h}$ or $48 \mathrm{~h}$ of challenge. Fixed lungs were visualized under stereo microscope 
(Leica MZ 16F; Leica Microsystems, Wetzlar, Germany) using a $1 \times$ lens. Red blood cells were counted after $48 \mathrm{~h}$ at $\times 40$.

\section{Statistical analysis}

Statistical analysis was performed using Student's $t$ test and Fisher's exact test. Results are expressed as means $\pm \mathrm{SE}$.

\section{RESULTS}

\section{ErbB2 transformation requires p130Cas}

To investigate the relevance of p130Cas in ErbB2 transformation, we transiently transfected NIH3T3 cells with transforming ErbB2 and two different p130Cassilencing vectors and performed a focus formation assay. p130Cas silencing resulted in $80 \%$ reduction of the protein (Fig. 1A, left panel) and up to $55 \%$ reduction in focus forming units (Fig. $1 A$, right panel), thus indicating that ErbB2 transformation in NIH3T3 cells is strongly dependent on p130Cas expression. Moreover, p130 Cas $^{-/-}$mouse embryo fibroblasts (MEFs) derived from knockout animals (14), their $+/+$ wild-type counterparts, and the $-/-$ MEFs reconstituted with the p130Cas full-length (FL) protein were infected with retroviruses expressing human ErbB2 oncogene (Fig. 1B, left panel), and soft agar assays were performed. Soft agar colonies were counted within $2 \mathrm{wk}$ from plating. Notably, wild-type MEFs did not form colonies in soft agar, and they acquired the ability to grow in anchorage-independent conditions only following transformation with ErbB2. On the contrary, ErbB2 infection of p130Cas ${ }^{-/-}$MEFs was not sufficient to drive colony formation. Consistently, reexpression of the FL protein in ErbB2 infected p130Cas ${ }^{-/-}$cells rescued the capacity of forming colonies in soft agar (Fig. $1 \mathrm{~B}$, left panel), indicating that p130Cas is required for anchorage-independent growth induced by ErbB2.

p130Cas down-regulation was performed in N202-1A breast cancer epithelial cells, which are derived from mammary tumors overexpressing NeuN (17). We generated cells expressing doxycycline-inducible p130Cas shRNAs or control scramble shRNA sequences. Within $48 \mathrm{~h}$ of doxycycline treatment, p130Cas expression was effectively silenced of $\sim 75 \%$ compared to cells infected with scramble sequences, reaching the lowest level at
A

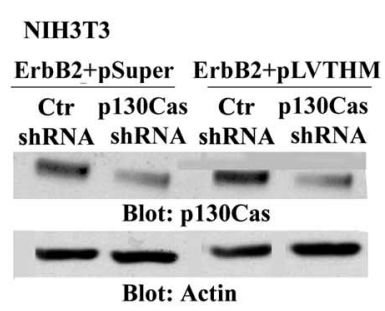

B Cas-I-MEF

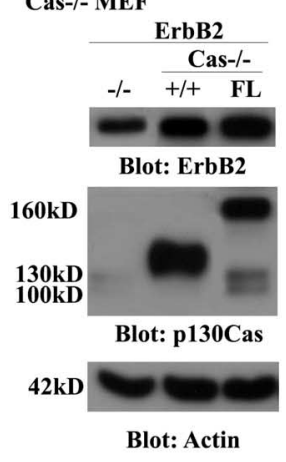

\begin{tabular}{lc} 
DNA & No. of f.f.u. \\
\hline ErbB2 & $94+/-4$ \\
ErbB2 + pSuper-Ctr-shRNA & $80+/-3$ \\
ErbB2 + pSuper-p130Cas-shRNA & $42+/-3$ \\
ErbB2 + pLVTHM-Ctr-shRNA & $103+/-6$ \\
ErbB2 + pLVTHM-p130Cas-shRNA & $45+/-2$
\end{tabular}

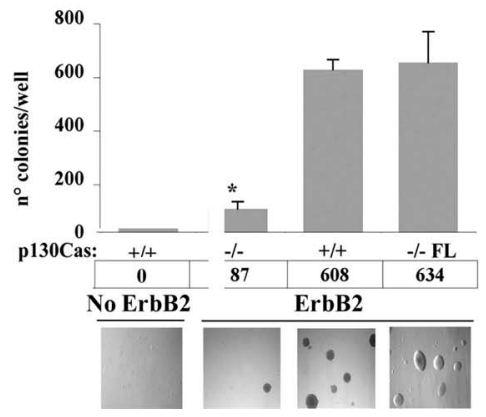

C
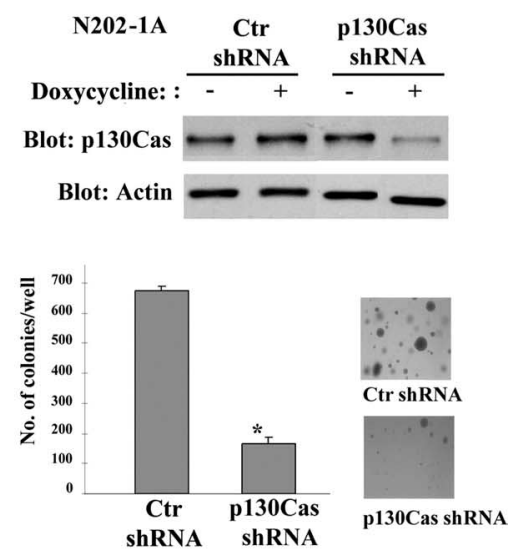

Figure 1. p130Cas expression is essential for ErbB2 anchorage-independent growth. A) Left panel: total cell extracts from NIH3T3 transiently cotransfected with activated ErbB2 and shRNA vectors (pSuper Retro, pLVTHM) expressing the scramble control (Ctr shRNA) or the silencing sequence against p130Cas (p130Cas shRNA) were blotted with p130Cas antibodies. Actin was used as a loading control. Right panel: focus formation assays were performed with cells transfected as indicated above. As further control, NIH3T3 cells transfected with ErbB2 alone were used. Average \pm sD number of foci was calculated from 3 independent assays performed in triplicates; images of a representative experiment are shown on the right.

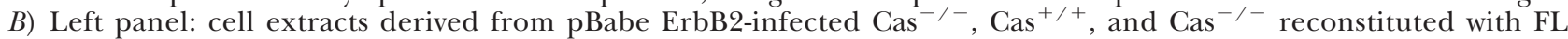
p130Cas-GFP MEFs were blotted with antibodies to ErbB2 (185 kDa, top panel), p130Cas (middle panel), and actin as loading control (bottom panel). MW markers are shown at left. Note that the FL (p130Cas-GFP) runs at $160 \mathrm{kDa}$. Right panel: uninfected $\mathrm{Cas}^{+/+}$and $\mathrm{Cas}^{-/-}, \mathrm{Cas}^{+/+}$and Cas FL MEFs infected with pBabe-ErbB2 were subjected to soft agar assays. Statistically significant quantification of visible colonies and representative images taken at 2 wk of growth are shown. Experiments were repeated 5 times in triplicate. C) Top panel: N202-1A cells were infected with lentiviruses expressing scramble shRNA (Ctr shRNA) or p130Cas shRNAs (p130Cas shRNA) and treated or not with doxycycline for $72 \mathrm{~h}$. Total cell extracts were blotted with p130Cas antibodies and actin for loading control. Bottom panel: same cells were subjected to soft agar assays. Quantification of visible colonies in 3 independent experiments performed in triplicate and representative images taken at 3 wk of growth are shown. $* P<0.01$. 
$72 \mathrm{~h}$ (Fig. $1 C$, top panel). In soft agar assays, p130Cassilenced cells demonstrated a 4-fold decrease in the number of colonies (Fig. 1C, bottom panel), indicating that the down-regulation of p130Cas is sufficient to significantly impair anchorage-independent growth of tumor cells overexpressing the ErbB2 proto-oncogene.

\section{p130Cas silencing impairs in vivo tumor growth}

To investigate the relevance of p130Cas in ErbB2dependent tumor growth, N202-1A cells expressing doxycycline-inducible p130Cas shRNAs, control scramble shRNA sequences, or silenced cells reconstituted by transiently transfection with human p130Cas full-length cDNA to recover protein expression (Fig. 2A) were orthotopically injected into the mammary fat pad of FVB-NeuN mice. Mice were provided with doxycyclinesupplemented drinking water. p130Cas silenced cells gave rise to tumors only in 6 of 10 mice, and these tumors were significantly smaller in size compared to scramble and reconstituted cells (Fig. 2B).

As an alternative approach, to assess the role of p130Cas in the growth of ErbB2-dependent spontaneous tumors, Balbc NeuT mice (26), which develop focal mammary tumors at 8 wk of age, were used. Control or p130Cas-stabilized siRNAs were locally delivered by intranipple injection (24) in the fourth mammary gland of 12-wk-old mice, and the treatment was repeated weekly for $5 \mathrm{wk}$. Mice were sacrificed at wk 18 . Whole-mount analysis showed that mammary glands treated with p130Cas siRNAs developed significantly less severe lesions than glands injected with control siRNAs (Fig. 2C). Moreover, Western blot analysis of mammary gland extracts revealed that p130Cas was effectively knocked down in vivo by specific siRNA injection, and its down-regulation correlated with decreased Fak phosphorylation, Src and Akt activation, and reduced level of cyclin D1 (Fig. 2D). Thus, these experiments demonstrate that selective knockdown of p130Cas impairs orthotopic ErbB2-dependent tumor formation. In addition, p130Cas silencing during the progression of spontaneous ErbB2 tumors is sufficient to significantly decrease their growth.

\section{In ErbB2-transformed cells, Fak and c-Src signaling is dependent on p130Cas, and p130Cas is required for} the assembly of a macromolecular complex with ErbB2, Fak and c-Src

It is known that c-Src and Fak associate to p130Cas and that c-Src activation accounts for p130Cas and Fak phosphorylation, creating signaling platforms that regulate receptor tyrosine kinase activity and downstream signaling (8). Analysis of cell extracts revealed that the
A

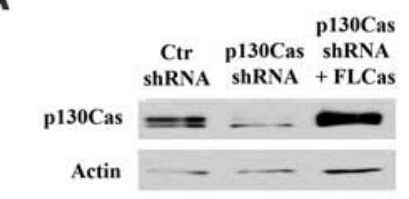

B
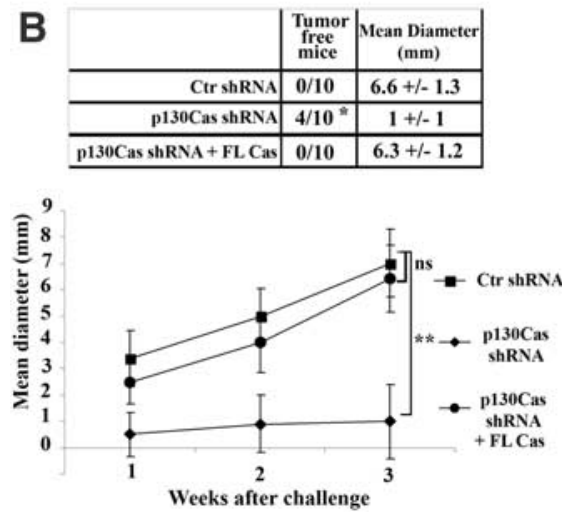

C

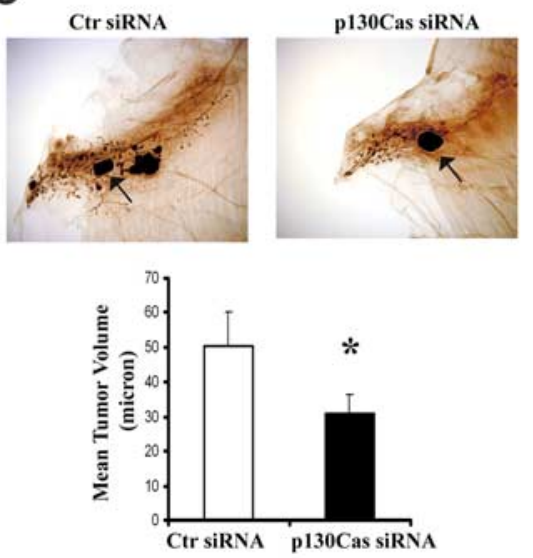

D

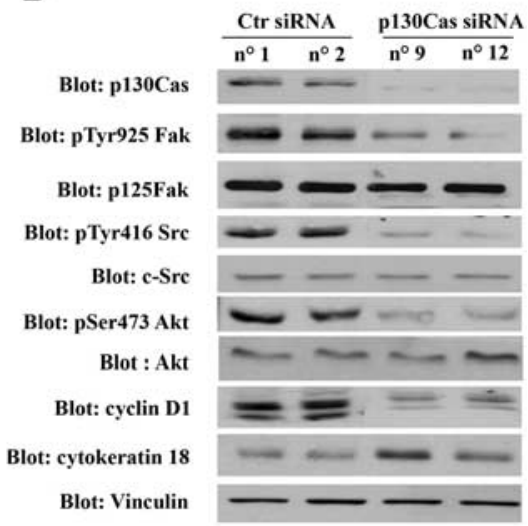

Figure 2. p130Cas is required for in vivo ErbB2 tumorigenesis. A) Total cell extracts from infected N202-1A cells expressing scramble shRNA (Ctr shRNA), p130Cas shRNAs (p130Cas shRNA), and silenced cells reconstituted with the human full-length p130Cas (p130Cas shRNA+hFL Cas) were blotted with antibodies to p130Cas and actin as loading control. B) Cells as in A were orthotopically injected into FVB-NeuN mice, and tumor diameter was measured

$1 \times /$ wk within $21 \mathrm{~d}$. Top panel: quantification of number and size of tumors at $21 \mathrm{~d}$ after injection. $* P<0.05$ (Fisher's exact test). Bottom panel: mean diameter is reported on the $y$ axis. $* * P<0.001$; ns, $P=0.2$ (Student's $t$ test). $C$ ) Intranipple injection was performed in BalbC-NeuT female mice. Control (Ctr siRNA) or p130Cas-stabilized siRNA (p130Cas siRNA) was injected $1 \times /$ wk for $5 \mathrm{wk}$ starting from wk 12. Top panels: whole-mount analyses of fixed mammary gland at wk 18 . The gland is composed of a tree-like structure of branching ducts. Small lesions that have histological aspects of a solid carcinoma are visible. Black arrows indicate the lymph node. Ctr siRNA picture shows larger lesions on the right of the lymph node. Bottom panel: histogram shows the mean tumor volume measured from 2 independent experiments with 8 mice/group. $* P<0.0329$ (2-tailed $P$ value). $D$ ) Protein extracts were prepared from mammary glands from Ctr (no. 1, no. 2) and p130Cas siRNA intranipple-injected mice (no. 3, no. 4). Western blot analysis was performed with antibodies to p130Cas, pTyr925Fak, p125Fak, pTyr416Src, c-Src, pSer473Akt, Akt, cyclinD1, cytokeratin 18, and vinculin as loading control. 
phosphorylation of pTyr416 Src and pTyr925 Fak, a known c-Src substrate, was reduced by $75 \%$ in p130Cas silenced N202-1A cells compared to control and to human p130Cas reconstituted cells (Fig. 3A). Thus, in cells transformed by ErbB2, p130Cas controls c-Src activation and the efficient phosphorylation of Fak.

When control N202-1A cell extracts were immunoprecipitated with p130Cas antibodies, ErbB2, Fak, and c-Src were found to coimmunoprecipitate with p130Cas (Fig. 3B). To assess the specific contribution of p130Cas in the complex formation, cell extracts of control and p130Cas-silenced N202-1A cells were immunoprecipitated with antibodies specific for ErbB2, Fak and c-Src (Fig. 3C). Interestingly, in p130Cas silenced cells recip- rocal immunoprecipitation experiments showed that the association of ErbB2 with Fak and c-Src was markedly reduced as well as the complex between Fak and c-Src (Fig. 3C), indicating that down-regulation of p130Cas profoundly affects the assembly of the macromolecular complex. Tyrosine phosphorylation could be relevant for the association of the molecules. Therefore, to assess the contribution of kinase activity in complex formation, pharmacological inhibitors for cSrc (SU6656), Fak (PT573228), and ErbB2 (Lapatinib) were used. The capacity of each inhibitor to inhibit Src and Fak activation and ErbB2 phosphorylation was tested (Fig. 3D). The association of ErbB2, p130Cas, Fak, and c-Src was evaluated on control N202-1A cells

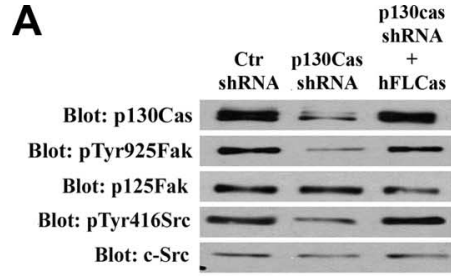

B

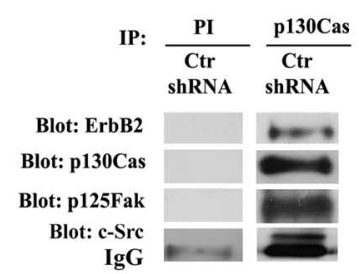

$\mathbf{E}$

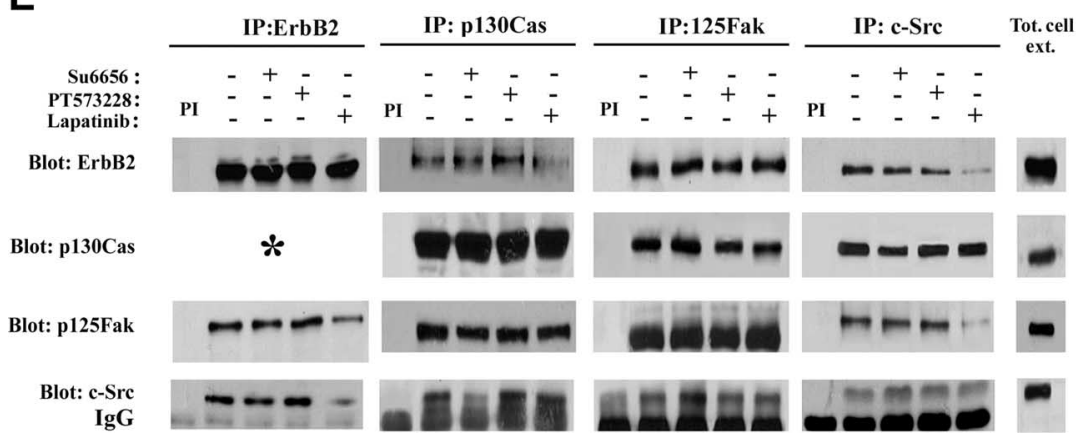

C
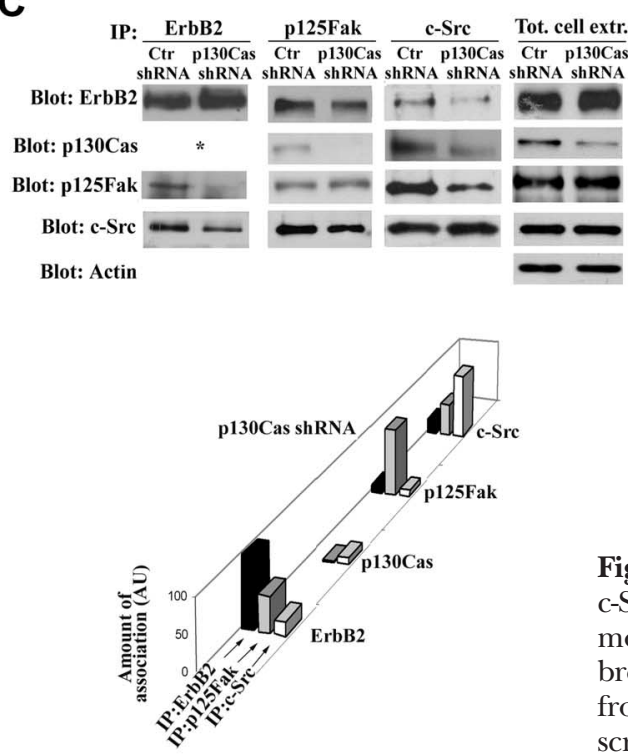

D
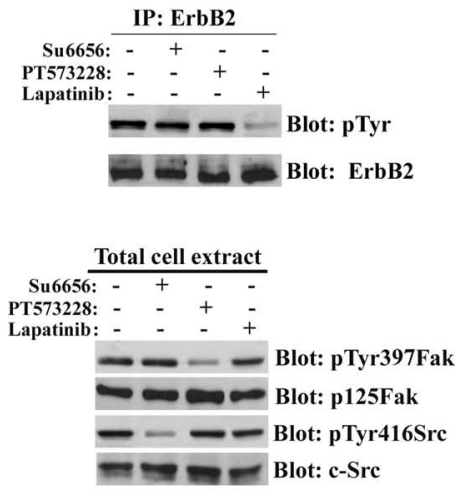

Figure 3. p130Cas is required for Fak and c-Src signaling and takes part of a macromolecular complex in ErbB2-transformed breast cancer cells. A) Total cell extracts from N202-1A-infected cells expressing scramble shRNA (Ctr shRNA), p130Cas shRNAs (p130Cas shRNA), or silenced cells reconstituted with the human fulllength p130Cas ( 130 Cas shRNA+hFL) treated with doxycycline for $72 \mathrm{~h}$ were evaluated by Western blot analysis for pTyr925Fak, p125Fak, pTyr416Src, cSrc, and vinculin as loading control. Results are representative of 3 independent experiments. $B$ ) Cell extracts from N202-1A cells expressing scramble shRNA (Ctr shRNA) in the presence of doxycycline were immunoprecipitated with isotype-specific antibodies (PI) or $\mathrm{mAbs}$ for p130Cas. Immunoprecipitates were blotted with antibodies to ErbB2, p130Cas, p125Fak, and cSrc. C) N202-1A cells expressing scramble shRNA (Ctr shRNA) or p130Cas shRNAs (p130Cas shRNA) in the presence of doxycycline were immunoprecipitated with mAbs for ErbB2, p125Fak, and c-Src. Top panel: immunoprecipitates and total cell extracts were blotted with antibodies to ErbB2, p130Cas, p125Fak, c-Src, and actin for loading control. Bottom panel: illustrative graph visualizing changes in association of the proteins in the different immunoprecipitates. Amount of associated protein in p130Cas-silenced cells is reported as the percentage of that found in control cells. *Note that in the ErbB2 immunoprecipitates, p130Cas was not detected, probably due to inaccessibility of the epitope. $D$ ) Top panel: total cell extracts from N202-1A cells untreated or treated with $10 \mu \mathrm{M}$ c-Src inhibitor (SU6656), $10 \mathrm{nM}$ p125Fak inhibitor (PT573228) and with $0.5 \mu \mathrm{M}$ ErbB2 inhibitor (Lapatinib) were immunoprecipitated with ErbB2 antibodies. Immunoprecipitates were evaluated by Western blot for phosphorylation of ErbB2 (pTyr). Bottom panel: Total cell extracts were blotted for pTyr397Fak, p125Fak, pTyr416Src, and c-Src. Results are representative of 3 independent experiments. E) Cell extracts from N202-1A cells untreated or treated as in $D$ were immunoprecipitated with isotype-specific antibodies (PI) and with mAbs for ErbB2, p130Cas, p125Fak, and c-Src. Immunoprecipitates were blotted with antibodies to ErbB2, p130Cas, p125Fak, and c-Src. *As in C, in the ErbB2 immunoprecipitates, p130Cas was not detected, probably due to inaccessibility of the epitope. 
untreated or treated with each of the three inhibitors by immunoprecipitation with antibodies specific for ErbB2, p130Cas, Fak, and c-Src and reciprocal Western blot analysis. In cells treated with Lapatinib, reciprocal immunoprecipitations show that ErbB2 kinase activity was required for its immunoprecipitation with c-Src, and for its association with p130Cas, indicating that the inhibition of the catalytic activity of ErbB2 impairs its association with p130Cas and c-Src. The treatment with the c-Src inhibitor (SU6656) mainly impaired c-Src association with p130Cas, but it did not affect the association of ErbB2 and p130Cas. When Fak was inhibited with PT573228, the relative immunoprecipitation levels did not change, suggesting that the catalytic activity of Fak is not relevant for the assembly of the macromolecular complex (Fig. 3E). Taken together, these data indicate that the major components driving the formation of the complex are p130Cas and active ErbB2, while active c-Src is required for its association with p130Cas. A role for Fak as an adaptor cannot be excluded.

In agreement with the data reported in N202-1A cells, ErbB2, c-Src, and Fak were found in p130Cas immunoprecipitates in FL-expressing MEFs, and the amounts of ErbB2 and Fak were reduced in c-Src immunoprecipitates in p130Cas ${ }^{-/-}$MEFs (Supplemental Fig. 1A). Consistently, when cell extracts from

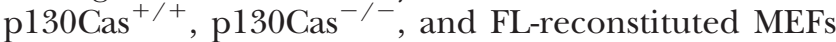
transformed by ErbB2 were analyzed; phosphorylation of pTyr925 Fak and pTyr416 Src was higher in p130 Cas ${ }^{+/+}$and FL-reconstituted cells compared to p130 $^{-1-}$ MEFs (Supplemental Fig. 1B).

In summary, these data indicate that in ErbB2transformed cells, c-Src and Fak signaling are dependent on the presence of p130Cas and that p130Cas is a key player for the assembly of a ErbB2/c-Src/Fak molecular complex.

\section{p130Cas is tyrosine phosphorylated on activation of ErbB2 and increases the extent of downstream signaling}

To study the effects of ErbB2 activation on p130Cas and downstream signaling, we used the human 10A.B2 cells expressing the ErbB2 chimera, which is activated by treatment with the synthetic ligand AP1510 (27). Extracts from 10A.B2 cells treated or not with AP1510 were immunoprecipitated with ErbB2 and p130Cas antibodies. As shown in Fig. 4A, ErbB2 and p130Cas phosphorylation started at $5 \mathrm{~min}$ of stimulation and persisted over time. Interestingly, c-Src activation was not detectable at $5 \mathrm{~min}$, but it occurred after 15 min of treatment. Moreover, treatment of cells with the c-Src inhibitor SU6656 did not prevent p130Cas phosphorylation (data not shown), indicating that the early p130Cas phosphorylation is dependent on ErbB2, but not on c-Src activity. 10A.B2 cells were infected with retroviral particles carrying p130Cas-GFP cDNA (10A.B2Cas) or the appropriate control (10A.B2mock) and analyzed for p130Cas levels (Fig. 4B). In addition, tyrosine phosphorylation analysis of GFP-p130Cas chimeric product and of endogenous p130Cas showed that both proteins are phosphorylated at comparable levels on 5 min of AP1510 treatment (Supplemental Fig. 2). Interestingly, in 10A.B2Cas cells in absence of AP1510, ErbB2 tyrosine phosphorylation was higher compared to 10A.B2mock cells, suggesting that high levels of p130Cas function as a priming event for a partial activation of the receptor (Fig. 4C).

In addition, 10A.B2mock and 10A.B2Cas cells were treated with AP1510, and cell extracts were analyzed for activation of c-Src, Akt and Erk1/2 MAPK, and Fak phosphorylation. p130Cas overexpression in 10A.B2 cells leads to increased activation of c-Src, Erk1/2, Akt, and to higher Fak phosphorylation over the mock cells, even in the absence of AP1510 (Fig. 4D). In 10A.B2Cas cells, p130Cas coimmunoprecipitates with ErbB2, Src, and Fak (data not shown), suggesting that these molecules associate also in this cellular model.

These data indicate that increased levels of p130Cas are sufficient to induce tyrosine phosphorylation of ErbB2 and to strengthen activation of downstream signaling pathways.

\section{p130Cas overexpression confers invasive properties to activated ErbB2 in a three-dimensional (3-D) model of epithelial acini}

In 3-D cultures, 10A.B2 cells forms acini-like spheroids that recapitulate the terminal ductal lobular unit of human adult breast. Activation of ErbB2 disrupts the morphogenetic process giving rise to multiacinar, noninvasive structures, mimicking the early stages of breast cancer (27).

When plated on a 3-D basement membrane (Matrigel/collagen I 1:1), 10A.B2mock cells formed normal acini (Fig. 5Aa, $\boldsymbol{b}$ ), which in the presence of AP1510 ligand, converted into multiacinar structures (Fig. $5 \mathrm{Ac}$, d). In contrast, 10A.B2Cas cells gave rise to multiacinar structures (Fig. 5Ae, $f$ ) that were positive for the proliferating cell antigen Ki-67 (Supplemental Fig. 3). Further, their formation was prevented by treatment with the ErbB2 inhibitor lapatinib, suggesting that p130Casdependent partial activation of ErbB2 may account for multiacinar phenotype (Supplemental Fig. 4). These data indicate that increased levels of p130Cas, in 3-D cultures, promote proliferation and multiacinar formation. Interestingly, when 10A.B2Cas cells were treated with AP1510, $50 \%$ of the large multiacinar structures showed invasive protrusions (Fig. $5 \mathrm{Ag}, h$; arrows), which, as shown by time-lapse microscopy (Fig. $5 B$, bottom panels), originated from the bulk multiacinar structure. These protrusions were not detectable either in untreated 10A.B2Cas cells or in mock cells stimulated with AP1510 (Fig. 5B, top panels). To determine whether invasion was associated with breakdown of the basement membrane, 3-D acini were stained for lami- 
A

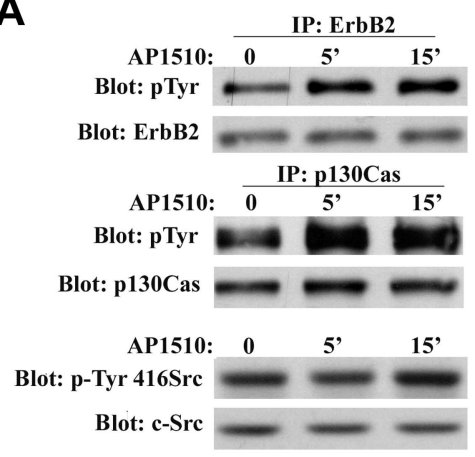

B

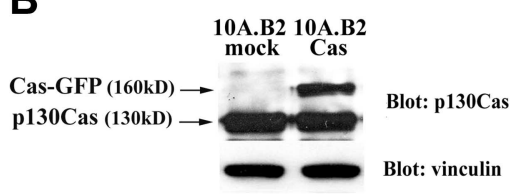

C

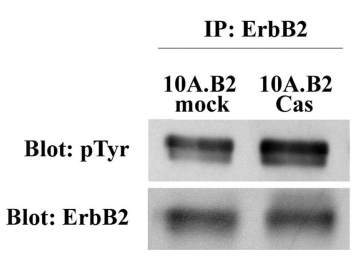

D

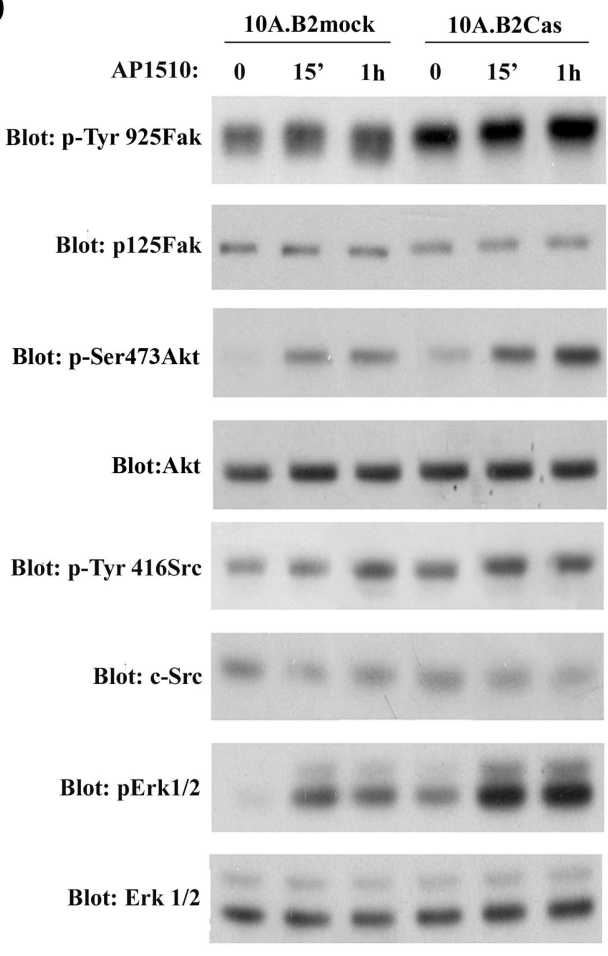

$\square$ 10A2.B2 mock $\quad$ 10A2.B2 Cas
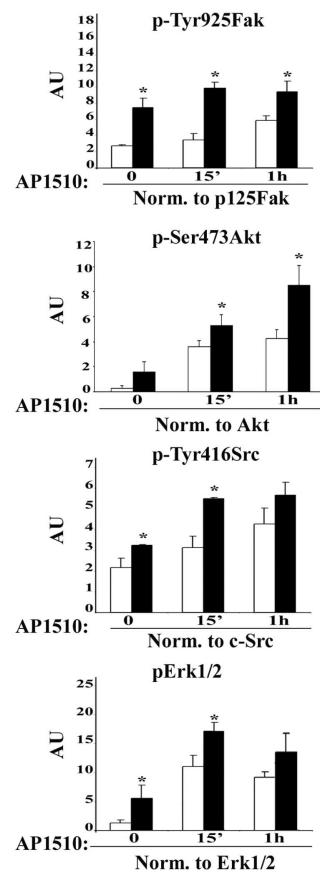

Figure 4. p130Cas is phosphorylated on ErbB2 activation and regulates the extent of ErbB2 downstream signaling. A) $10 \mathrm{~A} . \mathrm{B} 2$ cells stimulated at different times with $1 \mu \mathrm{M}$ AP1510 were immunoprecipitated with mAbs directed to the cytoplasmic domain of ErbB2 (top panel) and to p130Cas (middle panel). ErbB2 and p130Cas immunoprecipitates were blotted with antibodies to phosphotyrosine (pTyr) and reblotted with antibodies to ErbB2 and p130Cas, respectively. Total cell extracts were blotted with antibodies to pTyr416Src and normalized for total c-Src protein level (bottom panel). B) Total cell extracts of 10A.B2mock and 10A.B2Cas cells were probed with mAbs for p130Cas and for vinculin as a loading control. Arrows indicate the molecular mass of endogenous p130Cas (130 kDa) and of p130Cas-GFP fusion protein (160 kDa). C) Cell extracts from 10A.B2mock and 10A.B2Cas starved cells were immunoprecipitated with mAbs directed to the cytoplasmic domain of ErbB2, and immunoprecipitates were blotted with anti-phosphotyrosine antibodies and reblotted with antibodies against ErbB2. D) Left panels: Western blot analysis of total cell extracts of 10A.B2mock cells and 10A.B2Cas cells treated for different times with AP1510. Cell extracts were probed with antibodies to pTyr925Fak, p125Fak, pSer473Akt, Akt, pTyr416Src, c-Src, pErk1/2, and Erk1/2. Right panels: Quantification analysis and significance of 3 independent experiments. $* P<0.05$.

nin V, a major component of basal membranes. A continuous layer of laminin $\mathrm{V}$ surrounded 10A.B2mock treated acini (Fig. $5 C$ b). On the contrary, in the 10A.B2Cas treated acini, laminin staining showed points of breakage corresponding to invading cells (Fig. $5 C d, D e-h$; arrows). For studying molecular mechanisms related to the invasive phenotype, transwell invasion assays were performed through a layer of Matrigel-coated membranes. 10A.B2Cas, in the presence of ErbB2 activation, invaded 2 times more compared to control cells (Fig. 6A). In the same experimental set, Rac1 activity measured by pulldown assay was markedly increased in 10A.B2Cas, compared to mock cells, on ErbB2 activation (Fig. 6B). Consistently, gelatin zymography assays showed that the matrix metalloproteinase 9 (MMP9) activity was higher on concomitant activation of ErbB2 and p130Cas overexpression (Fig. 6C). Taken together, these results clearly indicate that, while ErbB2 activation does not give rise to invasion of basement membranes (28), concomitant activation of ErbB2 and overexpression of p130Cas lead to the acquirement of invasive properties in human breast cancer epithelial cells.
p130Cas controls motility, invasion, and lung colonization of ErbB2-transformed cells

To evaluate whether the down-regulation of p130Cas is sufficient to affect cell migration and invasion of ErbB2transformed cells, the N202-1A cells silenced with p130Cas-shRNAs were analyzed in migration and invasion assays. p130Cas knockdown in N202-1A cells induced a 2-fold decrease in migration (Fig. 7A), as well as a significant reduction of invasion (Fig. $7 B$ ), compared to cells infected with scramble sequences.

Consistently, in p130Cas ${ }^{-/-}$MEFs transformed by ErbB2, the absence of p130Cas resulted in the inability to migrate and invade, which was rescued by re-expression of FL p130Cas (data not shown). Indeed, ErbB2-

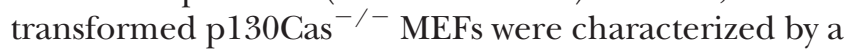
round morphology and a cortical actin distribution (Supplemental Fig. 5A), while the p130 $\mathrm{Cas}^{+/+_{-}}(B)$ and the FL-reconstituted cells $(C)$ became elongated with organized actin stress fibers. Taken together, these data indicate that ErbB2, in order to promote migration and invasion of p130Cas ${ }^{-/-}$MEFs, requires p130Cas to 
A

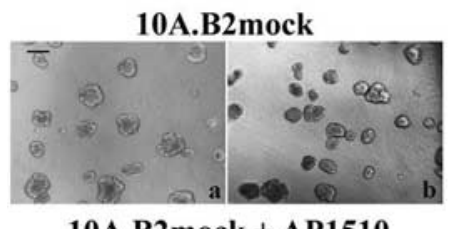

10A.B2mock + AP1510

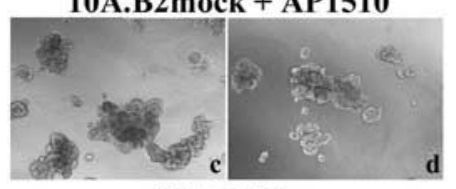

10A.B2Cas

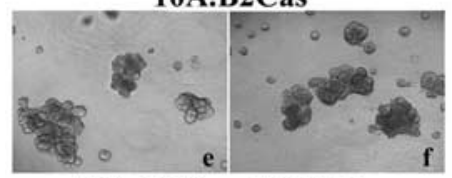

10A.B2Cas +AP1510

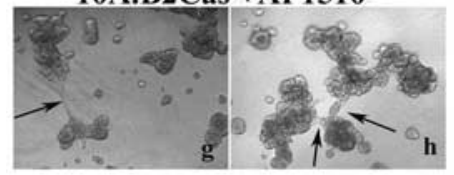

D

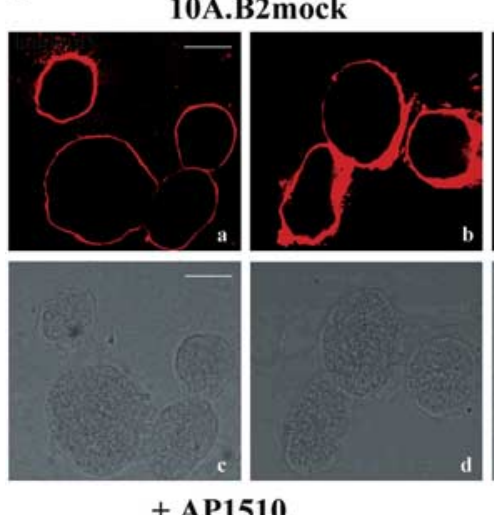

+ AP1510

B
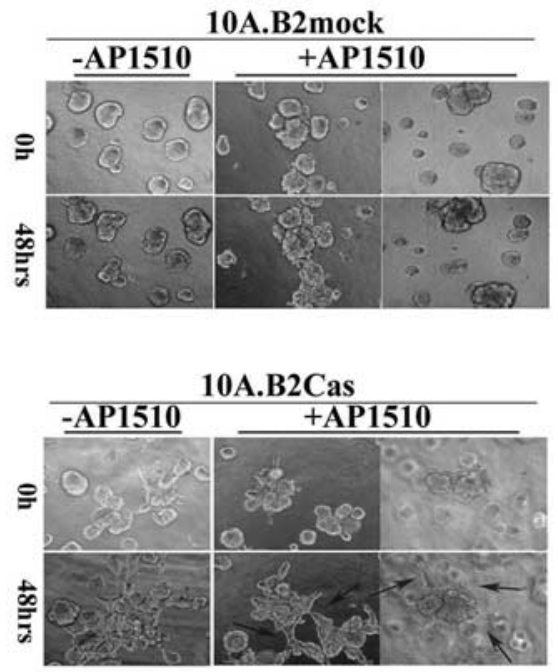

Figure 5. p130Cas overexpression and ErbB2 activation induce invasion in 3-D cultures. A) 10A.B2mock $(a-d)$ and 10A.B2Cas cells $(e-h)$ were grown in Matrigel/collagen I (1:1) for $12 \mathrm{~d}$ and then left unstimulated $(a, b, e, f)$ or stimulated for $4 \mathrm{~d}$ with AP1510 $(c, d, g, h)$. Images of acinar structures were taken at d 16. Two representative fields of 3 independent experiments are shown. Arrows indicate invasive protrusions. $B$ ) Live images of d 14 acinar structures formed by 10A.B2mock (top panels) and 10A.B2Cas cells (bottom panels) unstimulated or stimulated for $48 \mathrm{~h}$ with AP1510. Arrows indicate invasive structures. C) Laminin staining (red) of d 16 acini unstimulated $(a, c)$ or stimulated for $4 \mathrm{~d}$ with $\mathrm{AP} 1510(b, d)$. Only 10A.B2Cas stimulated cells $(d)$ show laminin breakpoints (arrows). D) Representative confocal images of laminin stained 10A.B2mock $(a, b)$, and 10A.B2Cas cells $(e, f)$ stimulated for $4 \mathrm{~d}$ with AP1510. Laminin breakpoints are indicated by ar-

rows $(e, f)$. Phase contrast images are shown $(c, d, g, h)$. Images were taken at $\times 20 / 0.7$ IMM with a Leica TCS-SP5 II confocal microscope and analyzed with LASAF software. Scale bars $=100 \mu \mathrm{m}(C) ; 50 \mu \mathrm{m}(D)$.

induce proper downstream signaling and actin cytoskeleton organization.

To further explore the involvement of p130Cas in invasion, we challenged p130Cas in in vivo lung metastasis formation. Since breast cancer epithelial N202-1A allografts do not metastasize (data not shown), an experimental metastasis assay of lung colonization was performed. N202-1A cells expressing scramble or silencing sequences without or with reconstitution with human p130Cas were treated with doxycycline and injected into the tail vein of nude mice, which were provided with doxycycline-supplemented drinking water. After 5 wk, mice injected with p130Cas-silenced N202-1A cells showed a 70\% reduction in the number of lung metastases with respect to mice injected with scramble control cells or with p130Cas reconstituted cells (Fig. 7C).

To assess whether the impaired lung colonization of p130Cas-silenced cells was due to increased cell death, anoikis assays were performed. N202-1A cells silenced with p130Cas-shRNAs and the appropriate controls were detached and kept in suspension on $4 \%$ agarosecoated plates for $24 \mathrm{~h}$. Indeed, cleaved Caspase-3 was present only in the extracts of p130Cas knocked-down cells, indicating that these cells are more susceptible to apoptosis induced by cell detachment (Fig. $7 D$ ). In addition, to evaluate whether p130Cas silencing can induce defects in transmigration through the microvasculature, in vitro and in vivo extravasation assays were performed. N202-1A cells silenced with p130CasshRNAs and the controls were plated on a monolayer of HUVECs and left to transmigrate. p130Cas-silenced cells had impaired ability to transmigrate through the endothelial barrier, with a $40 \%$ decrease of migrating cells (Fig. 7E). In vivo, N202-1A cells expressing scramble, p130Cas shRNA, or reconstituted cells were injected into the tail vein of nude mice, and mice were sacrificed after 2 or $48 \mathrm{~h}$. At $2 \mathrm{~h}$, the same number of cells was detected in the microvasculature of lungs with all the cell lines used (data not shown). At $48 \mathrm{~h}$, when 
A
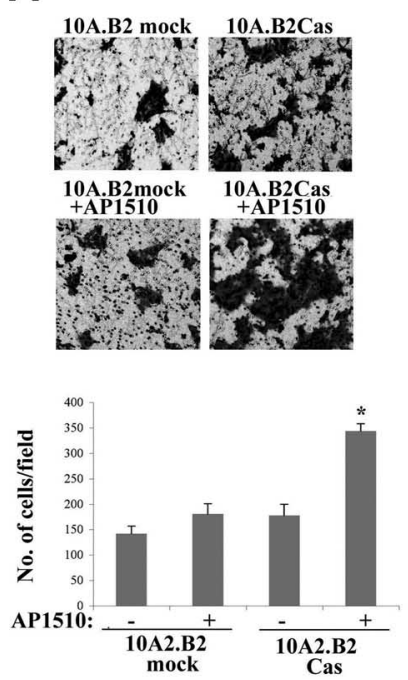

B

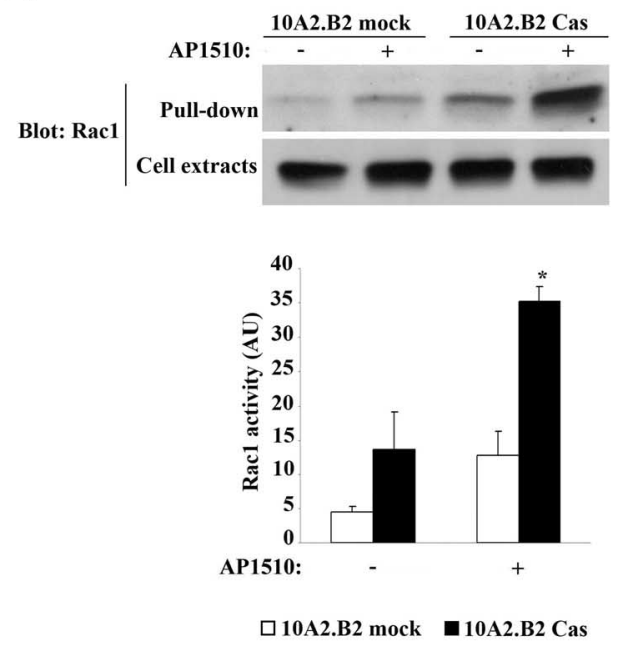

C
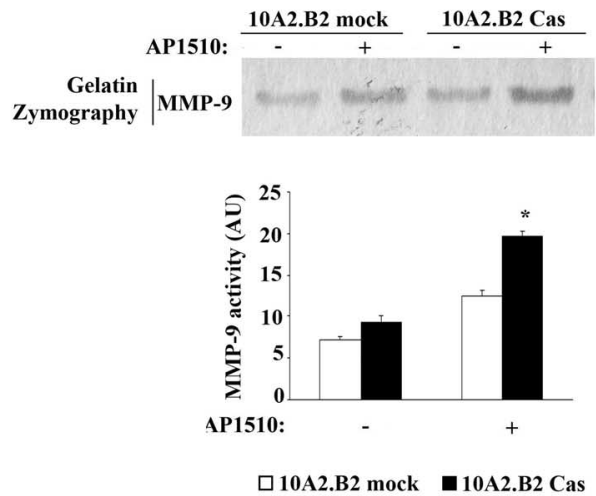

Figure 6. Cooperation of p130Cas and ErbB2 enhances Rac1 activity and MMP-9 secretion. A) 10A.B2mock and 10A.B2Cas cells stimulated with $1 \mu \mathrm{M}$ AP1510 were subjected to transwell invasion assays. After $48 \mathrm{~h}$, cells were

fixed and stained as described in Di Stefano et al. (21). Top panels: representative images of the bottom side of transwells. Bottom panel: quantification of cells that invaded through the Matrigel from 3 experiments performed in duplicate. B) 10A.B2mock and 10A.B2Cas cells were serum starved overnight and then untreated or treated with AP1510 for 15 min. Top panel, top blots: activated Rac1 was pulled down with the CRIB domain of PAK and detected by Western blot with mAb directed to Rac1. Bottom blots: total amount of Rac1. Bottom panel: quantification of active Rac1 from 3 independent experiments. C) 10A.B2mock and 10A.B2Cas cells were seeded onto 12-well plates and incubated for $48 \mathrm{~h}$ until they reached confluency. Cells were switched to serum-free medium without or with AP1510 for $18 \mathrm{~h}$, and medium was collected from each well and loaded onto $8 \%$ polyacrylamide gel impregnated with $0.1 \%$ gelatin, as described in Materials and Methods. Top panel: representative zymogram of MMP9 activity (92 kDa). Bottom panel: quantification of MMP9 activity from three independent experiments. $* P<0.05$.

cells have already extravasated, the number of p130Cassilenced cells detected in the lungs was significantly decreased compared to control or reconstituted cells (Fig. $7 F)$. Therefore, increased apoptosis and decreased extravasation are both implicated in the severe reduction of metastasis of p130Cas-silenced cells. In summary, these data strengthen the role of p130Cas as a major regulator of the invasive properties of ErbB2dependent breast cancer cells.

\section{DISCUSSION}

In this work, we demonstrate that p130Cas is required for ErbB2-mediated transformation and anchorageindependent growth; is phosphorylated on ErbB2 activation and is an essential transducer in ErbB2 signaling; and is necessary for driving migration and invasion of ErbB2-transformed cells. In addition, p130Cas silencing in breast cancer cells transformed by the ErbB2 proto-oncogene is sufficient to inhibit in vivo tumor growth and lung colonization.

\section{p130Cas is required for ErbB2-dependent transformation and tumor growth}

p130Cas is a key signaling molecule that conveys signals from many amplified or mutated oncogenes, such as ornithine decarboxylase, Ha-Ras, K-Ras, B-Raf, PTEN,
PIK3CA, c-Src, and ALK oncogenes $(14,29,30)$ by undergoing hyperphosphorylation and association with multiple signaling partners required for transformation. The data presented here show that the ErbB2 oncogene requires p130Cas as an essential transducer to drive foci formation and anchorageindependent growth. Indeed, we show that the reexpression of the FL protein is sufficient to promote growth of colonies in soft agar in p130Cas-null MEFs on ErbB2 transformation. Interestingly, when these cells were kept in suspension to mimic the anchorage-independent growth, a sustained activation of Akt was found in FL expressing cells (Supplemental Fig. 1C), likely accounting for their growth properties in soft agar. Thus, p130Cas is essential for triggering anchorage-independent growth on ErbB2 transformation, likely through the activation of survival mechanisms (7-9).

The relevance of p130Cas in tumor growth is also strengthened by in vivo studies, showing that p130Cas silencing is sufficient to inhibit in vivo ErbB2-dependent tumor formation. The growth of orthotopic tumors in FVB-NeuN mice and spontaneous ErbB2 tumors in FVB-NeuT mice was reduced. Interestingly, intranipple injection of p130Cas stabilized siRNAs in the mammary glands of mice in which spontaneous lesions were already present, was sufficient to silence p130Cas, to inhibit signaling and to reduce tumor lesions, indicating that p130Cas might be a potential therapeutic target for novel therapies using RNA interference. In summary, these different approaches clearly 
A

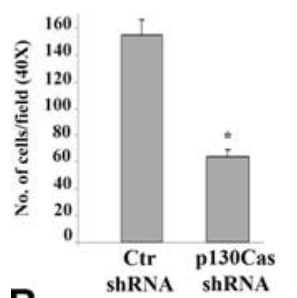

B

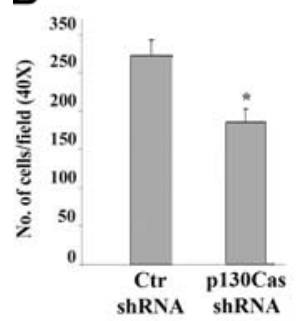

$\mathbf{F}$

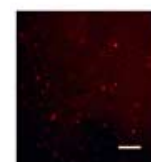

Ctr shRNA

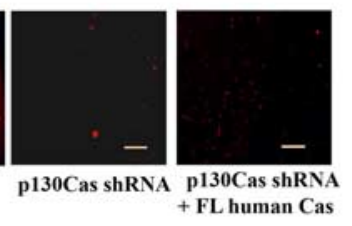

C

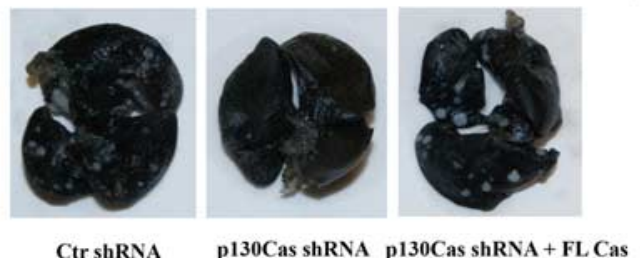

D

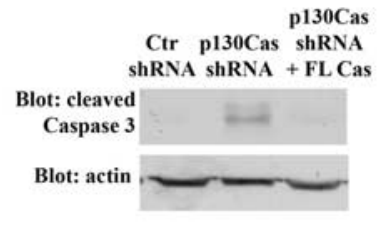

E
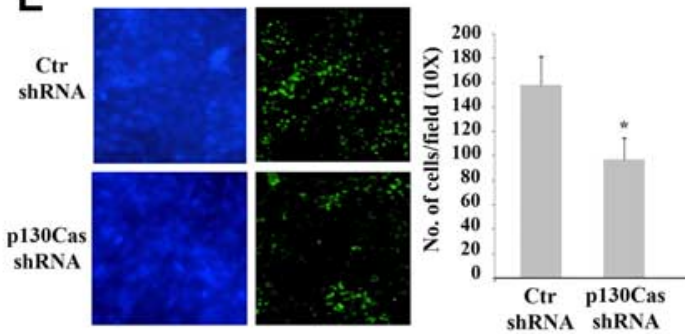

Figure 7. p130Cas expression is required for in vitro and in vivo migration and invasion of ErbB2 transformed cells. A) N202-1A cells infected with lentiviruses expressing scramble shRNA (Ctr shRNA) or p130Cas shRNAs (p130Cas shRNA) treated with doxycycline were subjected to transwell migration assays for $12 \mathrm{~h}$ in the presence of 20\% FBS. N202-1A cells in lower chambers were counted at $\times 40$. Graphs show average values of 3 independent experiments with statistical significance. ${ }^{*} P<0.05$. B) N202-1A cells as in $A$ were subjected to invasion assays on Matrigel-coated transwells for $72 \mathrm{~h}$ in the presence of $20 \%$ FBS. N202-1A cells in bottom chambers were counted at $\times 40$. Graphs show averages value of 3 independent experiments with statistical significance. $* P<0.05$. C) N202-1A epithelial cells infected with control shRNA (Ctr shRNA), p130Cas shRNA (p130Cas shRNA), or silenced cells reconstituted with human p130Cas cDNA (p130Cas shRNA+hFL Cas) were injected into tail veins of nude mice. Each experiment was performed with 5 mice/group. After 5 wk of doxycycline treatment, lungs of nude mice were stained with India ink, harvested, and fixed. Top panels: representative photographs of lungs from the 3 groups of mice. Bottom panel: quantification of surface lung nodules from 3 independent experiments with statistical significance. $* * P<0.01 . D$ ) Total cell extracts from Ctr, p130Cas shRNA, or p130Cas reconstituted N202-1A cells grown in suspension for 24 h were probed with antibodies to cleaved caspase 3 and normalized for actin for loading control. E) Left panels: Ctr and p130Cas shRNA N202-1A cells expressing GFP (see Materials and Methods) were plated on a previously seeded monolayer of HUVECs on transwells and allowed to migrate for $72 \mathrm{~h}$ in the presence of $20 \% \mathrm{FBS}$. Representative images of HUVEC monolayer stained with a blue fluorescent dye (left images), and of transmigrated cells after $72 \mathrm{~h}$ (right images) are shown. Right panel: quantification of migrated cells through HUVEC monolayer from 3 independent experiments with statistical significance. $* P<0.001 . F)$ Left panels: cells as in $C$ stained with fluorescent CellTracker red dye were injected into the tail vein of nude mice (5 mice/group). After 48 h, lungs were harvested and fixed in PFA 4\%. Representative images are shown. Right panel: Graph representing the quantification of fluorescent red cells present in the lung from 3 independent experiments with statistical significance. $* P<0.05$.

demonstrate that p130Cas is required for transformation and in vivo tumor growth driven by the ErbB2 oncogene.

\section{p130Cas is phosphorylated on ErbB2 activation and is an essential transducer in ErbB2 signaling}

At a mechanistic level, we demonstrate that p130Cas is a component of a macromolecular complex, that includes ErbB2, Fak, and c-Src. Although previous studies have demonstrated that c-Src tyrosine kinase interacts specifically with ErbB2 $(31,32)$ and that Fak and c-Src are partners of p130Cas (8), these results point out the existence of a novel signaling platform, which brings together all these molecules, and in particular, p130Cas with ErbB2. The knockdown of p130Cas and the use of pharmacological inhibitors for ErbB2, c-Src, and Fak show that the association of the 4 molecules requires the presence of p130Cas, active ErbB2, and active c-Src. Interestingly, ErbB2 kinase activity is required for the assembly of the complex, but p130Cas and c-Src are associated independently of ErbB2 activation. c-Src kinase activity is also necessary for its association with p130Cas, but not for the complex between ErbB2 and p130Cas. These data suggest that the p130Cas scaffold might independently associate with c-Src and ErbB2 and that the association of the molecules into the complex with ErbB2 is finely tuned by tyrosine phosphorylation. It has recently been shown that c-Src association with ErbB2 is not dependent on its $\mathrm{SH} 2$ or 
SH3 domain or on the phosphorylation or kinase activity of the receptor (32). Since our data show that p130Cas silencing disrupts c-Src/ErbB2 interaction, the relevance of p130Cas in the complex formation might rely on its ability to promote the conformational changes that have been suggested to mediate c-Src/ ErbB2 association. However, in our experimental model, the recruitment of the p130Cas/c-Src complex to ErbB2 requires ErbB2 activity, implying that phosphotyrosine-dependent interactions are also relevant. We cannot exclude that other known ErbB2 binding partners such as the p85 subunit of PI3K (1), also participate to this complex.

To point out the role of p130Cas in ErbB2 signaling, we used an inducible ErbB2 chimera expressed in the human mammary epithelial 10A.B2 cells (20). In these cells, p130Cas is tyrosine phosphorylated within $5 \mathrm{~min}$ of ErbB2 induction. p130Cas is a known substrate of c-Src (33). However, on ErbB2 activation, its phosphorylation occurs prior to ErbB2-mediated c-Src activation and is not reduced by pharmacological c-Src inhibition. Thus, in ErbB2-transformed cells, the early tyrosine phosphorylation of p130Cas correlates with ErbB2 activation but not with c-Src activity, suggesting that c-Src is not involved in early p130Cas phosphorylation.

Interestingly, p130Cas silencing dramatically affects c-Src activation in N202-1A ErbB2-transformed cells. Consistently, overexpression of p130Cas cooperates with ErbB2 activation, leading to a further increase in c-Src induction and in Fak phosphorylation on the tyrosine 925, a known phosphorylation site for c-Src, indicating a role for p130Cas in promoting a positive loop leading to c-Src induction mediated by ErbB2. In addition to c-Src, Akt and Erk1/2 MAPK also activities are up-regulated by high levels of p130Cas. Therefore, p130Cas per se is a positive regulator of signaling, that associates and cooperates with active ErbB2, driving c-Src, Fak, Akt, and Erk1/2 MAPK activation (Fig. 8).
ErbB2 activation in 10A.B2 cells gives rise to multiacinar structures (27), reflecting the in vivo amplification and activation of ErbB receptors in breast epithelial cells at the onset of malignancy. Our data show that increased expression of p130Cas in the 10A.B2Cas cells is sufficient to generate multiacinar structures, likely through the enhanced basal activity of c-Src, Fak, and Erk1/2. These multiacini are positive for Ki-67 staining, indicating that p130Cas overexpression mediates reentry into the cell cycle, likely through activation of the signaling pathways reported above. Consistently, in our MMTV-p130Cas transgenic mice, overexpression of p130Cas induces mammary epithelial cell hyperplasia, characterized by increased activation of proliferation pathways (10).

\section{p130Cas is required for ErbB2-dependent migration, invasion and in vivo lung metastasis colonization}

ErbB2 activation in 10A.B2 cells does not drive invasion (27). In contrast when ErbB2 is switched on in the 10A.B2Cas cells, the proliferation program is shut off, as shown by reduced KI67 staining, and these growtharrested multiacinar structures show extensive protrusions. These multiacinar structures switch from a stationary to an invasive phenotype, as demonstrated by discontinuities in the laminin $\mathrm{V}$ staining corresponding to invading cells. Moreover, both Rac1 and MMP9 activities are markedly enhanced, indicating that the concomitant expression of p130Cas and active ErbB2 is important for activation of pathways leading to progression of ErbB2 epithelial transformed cells to an invasive phenotype.

p130Cas silencing is sufficient to inhibit migration and invasion in cells transformed by ErbB2. The impaired organization of actin stress fibers in ErbB2 transformed p130Cas-null MEFs could account for the

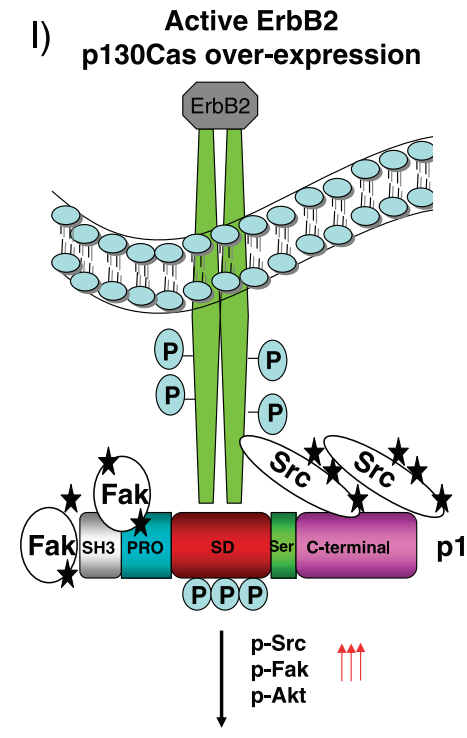

TRANSFORMATION AND INVASION

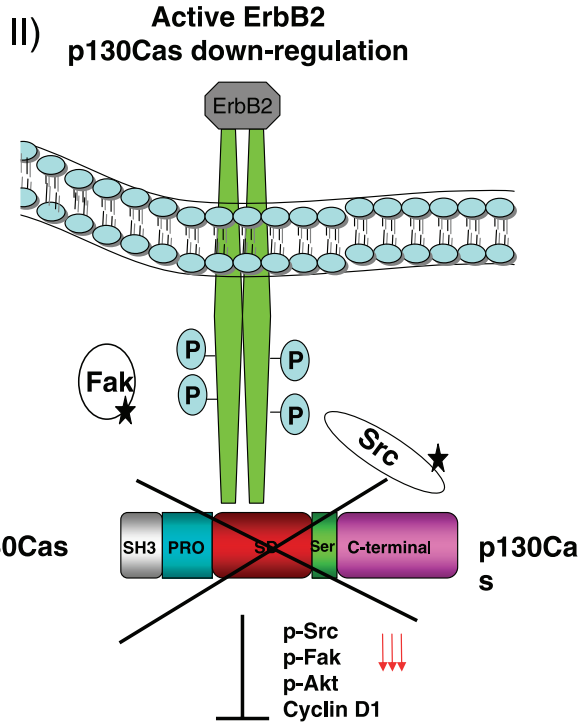

Figure 8. Model for the role of p130Cas in ErbB2 transformation and invasion. I) In breast cancer cells, high levels of p130Cas and concomitant ErbB2 activation result in enhanced phosphorylation of p130Cas and activation of c-Src, Fak, and Akt, leading to transformation and invasion. II) Conversely, low levels of p130Cas prevent the activation of downstream signaling, resulting in impaired tumorigenic properties. 
observed defects in the migratory/invasive machinery. p130Cas is involved in cell migration and invasion, mainly through its tyrosine phosphorylation by c-Src, and the assembly of a p130Cas/Crk/DOCK180 scaffold at lamellipodia and filopodia adhesion sites $(34,35)$, which ultimately leads to JNK activation and matrix metalloproteinases induction (36-38). In addition, in Fak-null MEFs, which are inhibited in migratory and invasive properties, even in the presence of oncogenic v-Src (39), the expression of p130Cas is sufficient to rescue invasion defects. Moreover, p130Cas has also been shown to enhance the capacity of oncogenic Src to invade through basement membranes in MEF-transformed fibroblasts (40). In mammary adenocarcinoma cell lines, ErbB2-dependent invasion has also been found to correlate with the coupling of p130Cas to Crk (41). Therefore, our data highlight the presence of a signaling platform, including ErbB2, Fak, c-Src, and p130Cas, where p130Cas is essential for driving migration and invasion.

Finally, p130Cas silencing efficiently prevents the lung metastasis colonization of ErbB2-transformed cells, by both increasing their susceptibility to apoptosis and impairing their ability to transmigrate through the endothelial barrier. This observation places p130Cas as a new player in the extravasation process, likely through its capacity to influence actin cytoskeleton remodeling.

In summary, this work outlines the essential role of p130Cas in ErbB2 tumor formation and in the acquirement of metastatic properties, providing evidences that p130Cas silencing might be a useful translational tool to prevent breast primary tumors to grow, to invade and to metastasize.

The authors thank Dr. Pier Giorgio Lollini (University of Bologna, Bologna, Italy) for the N202-1A cells, Dr. Nancy Hynes (Friedrich Miescher Institute, Basel, Switzerland) for human mutated ErbB2, Roberto Piva (University of Torino, Turin, Italy) for shRNA vectors, and Elisa Penna (Molecular Biotechnology Center, University of Torino) for the transmigration assays. M.P.C.-L. is supported by the Association for International Cancer Research (AICR), and A.T. received a short-term European Molecular Biology Organization fellowship for her stay in S.K.M.'s lab. This work was supported by AIRC, Associazione Italiana Ricerca Cancro, EU FP7 Metafight, Ministero Università e Ricerca, Programma di Ricerca di Rilevante Interesse Nazionale, Regione Piemonte-Progetti Sanità, Oncoprot, Piattaforma Staminali, Druidi, and Comitato Interministeriale per la Programmazione Economica, Compagnia San Paolo, Progetto Alfieri-Fondazione Cassa Risparmio Torino, Turin, Italy.

\section{REFERENCES}

1. Hynes, N. E., and MacDonald, G. (2009) ErbB receptors and signaling pathways in cancer. Curr. Opin. Cell Biol. 21, 177-184

2. Yarden, Y., Baselga, J., and Miles, D. (2004) Molecular approach to breast cancer treatment. Semin. Oncol. 31, 6-13

3. Hynes, N. E., and Lane, H. A. (2005) ERBB receptors and cancer: the complexity of targeted inhibitors. Nat. Rev. Cancer $\mathbf{5}$, 341-354

4. Matsuda, M., Mayer, B. J., Fukui, Y., and Hanafusa, H. (1990) Binding of transforming protein, P47gag-crk, to a broad range of phosphotyrosine-containing proteins. Science 248, 1537-1539
5. Reynolds, A. B., Kanner, S. B., Wang, H. C., and Parsons, J. T. (1989) Stable association of activated pp60src with two tyrosinephosphorylated cellular proteins. Mol. Cell. Biol. 9, 3951-3958

6. Sawada, Y., Tamada, M., Dubin-Thaler, B. J., Cherniavskaya, O., Sakai, R., Tanaka, S., and Sheetz, M. P. (2006) Force sensing by mechanical extension of the Src family kinase substrate p130Cas. Cell 127, 1015-1026

7. Bouton, A. H., Riggins, R. B., and Bruce-Staskal, P. J. (2001) Functions of the adapter protein Cas: signal convergence and the determination of cellular responses. Oncogene 20, 64486458

8. Defilippi, P., Di Stefano, P., and Cabodi, S. (2006) p130Cas: a versatile scaffold in signaling networks. Trends Cell Biol. 16, 257-263

9. O'Neill, G. M., Fashena, S. J., and Golemis, E. A. (2000) Integrin signalling: a new Cas 9 (t) of characters enters the stage. Trends Cell Biol. 10, 111-119

10. Cabodi, S., Tinnirello, A., Di Stefano, P., Bisaro, B., Ambrosino, E., Castellano, I., Sapino, A., Arisio, R., Cavallo, F., Forni, G., Glukhova, M., Silengo, L., Altruda, F., Turco, E., Tarone, G., and Defilippi, P. (2006) p130Cas as a new regulator of mammary epithelial cell proliferation, survival, and HER2-neu oncogene-dependent breast tumorigenesis. Cancer Res. 66, 46724680

11. Cabodi, S., Di Stefano, P. del Pilar Camacho Leal, M., Tinnirello, A., Bisaro, B., Morello, V., Damiano, L., Aramu, S., Repetto, D., Tornillo, G., and Defilippi, P. (2006) Integrins and Signal Transduction, Landes Bioscience, Austin, TX, USA

12. Mitra, S. K., and Schlaepfer, D. D. (2006) Integrin-regulated FAK-Src signaling in normal and cancer cells. Curr. Opin. Cell Biol. 18, 516-523

13. Stupack, D. G., Cho, S. Y., and Klemke, R. L. (2000) Molecular signaling mechanisms of cell migration and invasion. Immunol. Res. 21, 83-88

14. Honda, H., Oda, H., Nakamoto, T., Honda, Z., Sakai, R., Suzuki, T., Saito, T., Nakamura, K., Nakao, K., Ishikawa, T., Katsuki, M., Yazaki, Y., and Hirai, H. (1998) Cardiovascular anomaly, impaired actin bundling and resistance to Src-induced transformation in mice lacking p130Cas. Nat. Genet. 19, 361-365

15. Pylayeva, Y., Gillen, K. M., Gerald, W., Beggs, H. E., Reichardt, L. F., and Giancotti, F. G. (2009) Ras- and PI3K-dependent breast tumorigenesis in mice and humans requires focal adhesion kinase signaling. J. Clin. Invest. 119, 252-266

16. Muller, W. J., Sinn, E., Pattengale, P. K., Wallace, R., and Leder, P. (1988) Single-step induction of mammary adenocarcinoma in transgenic mice bearing the activated c-neu oncogene. Cell 54, $105-115$

17. Nanni, P., Pupa, S. M., Nicoletti, G., De Giovanni, C., Landuzzi, L., Rossi, I., Astolfi, A., Ricci, C., De Vecchi, R., Invernizzi, A. M., Di Carlo, E., Musiani, P., Forni, G., Menard, S., and Lollini, P. L. (2000) p185 (neu) protein is required for tumor and anchorageindependent growth, not for cell proliferation of transgenic mammary carcinoma. Int. J. Cancer 87, 186-194

18. Holbro, T., Beerli, R. R., Maurer, F., Koziczak, M., Barbas, C. F. 3rd, and Hynes, N. E. (2003) The ErbB2/ErbB3 heterodimer functions as an oncogenic unit: ErbB2 requires ErbB3 to drive breast tumor cell proliferation. Proc. Natl. Acad. Sci. U. S. A. 100, 8933-8938

19. Trono, D. (2003) Virology. Picking the right spot. Science 300, $1670-1671$

20. Cabodi, S., Moro, L., Baj, G., Smeriglio, M., Di Stefano, P. Gippone, S., Surico, N., Silengo, L., Turco, E., Tarone, G., and Defilippi, P. (2004) p130Cas interacts with estrogen receptor alpha and modulates non-genomic estrogen signaling in breast cancer cells. J. Cell Sci. 117, 1603-1611

21. Di Stefano, P., Damiano, L., Cabodi, S., Aramu, S., Tordella, L., Praduroux, A., Piva, R., Cavallo, F., Forni, G., Silengo, L., Tarone, G., Turco, E., and Defilippi, P. (2007) p140Cap protein suppresses tumour cell properties, regulating Csk and Src kinase activity. $E M B O ~ J . ~ 26,2843-2855$

22. Kim, I. Y., Yong, H. Y., Kang, K. W., and Moon, A. (2009) Overexpression of ErbB2 induces invasion of MCF10A human breast epithelial cells via MMP-9. Cancer Lett. 275, 227-233

23. Vanni, C., Ottaviano, C., Guo, F., Puppo, M., Varesio, L., Zheng, Y., and Eva, A. (2005) Constitutively active Cdc42 mutan confers growth disadvantage in cell transformation. Cell Cycle $\mathbf{4}$, $1675-1682$ 
24. Du, Z., Podsypanina, K., Huang, S., McGrath, A., Toneff, M. J., Bogoslovskaia, E., Zhang, X., Moraes, R. C., Fluck, M., Allred, D. C., Lewis, M. T., Varmus, H. E., and Li, Y. (2006) Introduction of oncogenes into mammary glands in vivo with an avian retroviral vector initiates and promotes carcinogenesis in mouse models. Proc. Natl. Acad. Sci. U. S. A. 103, 17396-17401

25. Cappello, P., Triebel, F., Iezzi, M., Caorsi, C., Quaglino, E., Lollini, P. L., Amici, A., Di Carlo, E., Musiani, P., Giovarelli, M., and Forni, G. (2003) LAG-3 enables DNA vaccination to persistently prevent mammary carcinogenesis in HER-2/neu transgenic BALB/c mice. Cancer Res. 63, 2518-2525

26. Boggio, K., Nicoletti, G., Di Carlo, E., Cavallo, F., Landuzzi, L., Melani, C., Giovarelli, M., Rossi, I., Nanni, P., De Giovanni, C., Bouchard, P., Wolf, S., Modesti, A., Musiani, P., Lollini, P. L., Colombo, M. P., and Forni, G. (1998) Interleukin 12-mediated prevention of spontaneous mammary adenocarcinomas in two lines of Her-2/neu transgenic mice. $J$. Exp. Med. 188, 589-596

27. Muthuswamy, S. K., Li, D., Lelievre, S., Bissell, M. J., and Brugge, J. S. (2001) ErbB2, but not ErbB1, reinitiates proliferation and induces luminal repopulation in epithelial acini. Nat. Cell Biol. 3, 785-792

28. Seton-Rogers, S. E., Lu, Y., Hines, L. M., Koundinya, M., LaBaer, J., Muthuswamy, S. K., and Brugge, J. S. (2004) Cooperation of the ErbB2 receptor and transforming growth factor beta in induction of migration and invasion in mammary epithelial cells. Proc. Natl. Acad. Sci. U. S. A. 101, 1257-1262

29. Auvinen, M., Paasinen-Sohns, A., Hirai, H., Andersson, L. C., and Holtta, E. (1995) Ornithine decarboxylase- and ras-induced cell transformations: reversal by protein tyrosine kinase inhibitors and role of pp130CAS. Mol. Cell. Biol. 15, 6513-6525

30. Ambrogio, C., Voena, C., Manazza, A. D., Piva, R., Riera, L., Barberis, L., Costa, C., Tarone, G., Defilippi, P., Hirsch, E., Boeri Erba, E., Mohammed, S., Jensen, O. N., Palestro, G., Inghirami, G., and Chiarle, R. (2005) p130Cas mediates the transforming properties of the anaplastic lymphoma kinase. Blood 106, 39073916

31. Kim, H., Chan, R., Dankort, D. L., Zuo, D., Najoukas, M., Park, M., and Muller, W. J. (2005) The c-Src tyrosine kinase associates with the catalytic domain of ErbB-2: implications for ErbB-2 mediated signaling and transformation. Oncogene 24, 7599-7607

32. Marcotte, R., Zhou, L., Kim, H., Roskelly, C. D., and Muller, W. J. (2009) c-Src associates with ErbB2 through an interaction between catalytic domains and confers enhanced transforming potential. Mol. Cell. Biol. 29, 5858-5871

33. Ruest, P. J., Shin, N. Y., Polte, T. R., Zhang, X., and Hanks, S. K. (2001) Mechanisms of CAS substrate domain tyrosine phosphorylation by FAK and Src. Mol. Cell. Biol. 21, 7641-7652

34. Burridge, K., and Wennerberg, K. (2004) Rho and Rac take center stage. Cell 116, 167-179

35. Zaidel-Bar, R., Kam, Z., and Geiger, B. (2005) Polarized downregulation of the paxillin-p130CAS-Rac1 pathway induced by shear flow. J. Cell Sci. 118, 3997-4007

36. Dolfi, F., Garcia-Guzman, M., Ojaniemi, M., Nakamura, H., Matsuda, M., and Vuori, K. (1998) The adaptor protein Crk connects multiple cellular stimuli to the JNK signaling pathway. Proc. Natl. Acad. Sci. U. S. A. 95, 15394-15399

37. Hsia, D. A., Mitra, S. K., Hauck, C. R., Streblow, D. N., Nelson, J. A., Ilic, D., Huang, S., Li, E., Nemerow, G. R., Leng, J., Spencer, K. S., Cheresh, D. A., and Schlaepfer, D. D. (2003) Differential regulation of cell motility and invasion by FAK. J. Cell Biol. 160, 753-767

38. Nakamoto, T., Yamagata, T., Sakai, R., Ogawa, S., Honda, H., Ueno, H., Hirano, N., Yazaki, Y., and Hirai, H. (2000) CIZ, a zinc finger protein that interacts with p130(cas) and activates the expression of matrix metalloproteinases. Mol. Cell. Biol. 20, $1649-1658$

39. Schlaepfer, D. D., Mitra, S. K., and Ilic, D. (2004) Control of motile and invasive cell phenotypes by focal adhesion kinase. Biochim. Biophys. Acta 1692, 77-102

40. Brabek, J., Constancio, S. S., Shin, N. Y., Pozzi, A., Weaver, A. M., and Hanks, S. K. (2004) CAS promotes invasiveness of Srctransformed cells. Oncogene 23, 7406-7415

41. Spencer, K. S., Graus-Porta, D., Leng, J., Hynes, N. E., and Klemke, R. L. (2000) ErbB2 is necessary for induction of carcinoma cell invasion by ErbB family receptor tyrosine kinases. J. Cell Biol. 148, 385-397

Received for publication February 22, 2010 Accepted for publication May 13, 2010. 